\title{
ENVEJECIMIENTO, ESTANCAMIENTO POBLACIONAL Y PERSPECTIVAS DEMOGRÁFICAS EN EXTREMADURA
}

\author{
Antonio Pérez Díaz y Felipe Leco Berrocal \\ Dpto. de Arte y Ciencias del Territorio, Universidad de Zaragoza \\ aperez@unex.es; fleco@unex.es
}

\begin{abstract}
Resumen: La situación de crisis que viene atravesando en los últimos años la economía mundial ha servido para despertar los viejos temores sobre las nefastas consecuencias socioeconómicas del envejecimiento demográfico. El recorte de las pensiones, el retraso de la edad de jubilación, la ampliación de los períodos de cotización y, en suma, la quiebra del estado del bienestar, han vuelto a convertirse en temas de actualidad después de permanecer plácida e interesadamente adormecidos durante la pretérita etapa de bonanza económica.

El presente artículo aborda algunos aspectos del envejecimiento demográfico en Extremadura, especialmente en lo concerniente a las causas de su temprano inicio, sus efectos sobre el estancamiento que padece la población regional y su previsible incidencia sobre el futuro demográfico de Extremadura.
\end{abstract}

Summary: The world economic crisis of the last few years has awakened the fears of the socioeconomic consequences of demographic aging. The need to cut down on pensions, to delay the age of retirement, to extend tax-paying periods and, in general, the collapse of the welfare state, are all problems that are again on the spotlight after having been deliberately left in the dark during the prosperous years.

This article discusses some aspects of demographic aging in Extremadura, such as the reasons why the phenomenon started earlier hear than in other areas of Spain, its effects on the stagnation of the population and its consequences for the demographic future of the region.

Keywords: Demographic aging, migration.

Recibido: 22-10-10. Aceptado: 3-11-11. 


\section{Introducción}

La crisis económica que afecta en la actualidad a las economías occidentales ha hecho retoñecer la preocupación de la opinión pública por temas que habían logrado pasar desapercibidos durante el período de bonanza económica. El recorte de las pensiones, el retraso en la edad de jubilación, la ampliación de los períodos de cotización, el copago sanitario y, en definitiva, la quiebra del estado del bienestar, han vuelto a convertirse en temas de actualidad después de permanecer plácida e interesadamente adormecidos durante años. En sus inicios, la crisis encontraba explicación en un tratamiento informativo que, enfocado casi exclusivamente desde perspectivas economicistas, giraba en torno a las dificultades del sistema financiero, las "hipotecas subprime", la falta de flexibilidad del mercado laboral o el pinchazo de la burbuja inmobiliaria. Poco a poco, sin embargo, estos planteamientos iniciales han ido derivando hacia nuevos derroteros, hasta el punto de considerar que, por encima de las dificultades económicas actuales y como agravante de éstas, "el principal problema de Europa en el siglo XXI es probablemente el demográfico”. En el caso de España, se afirma que harían falta entre 9 y 12 millones de personas menores de 30 años para mantener mínimamente, la "sostenibilidad de nuestro sistema público de pensiones y de sanidad a partir de la siguiente década" (Macarrón, 2010). Atendiendo a este tipo de afirmaciones, parece que el estado del bienestar en el que ha permanecido instalada la sociedad postindustrial, se tambalea. Lo que no queda del todo claro es si dicha situación obedece prioritariamente a problemas de orden económico o lo hace, sobre todo, a inconvenientes de tipo poblacional derivados del proceso de envejecimiento demográfico. En cualquier caso, ambas componentes, la económica y la demográfica, se encuentran indisolublemente unidas.

Fueron precisamente las transformaciones económicas propiciadas por la denominada primera revolución industrial (s. XVIII) las que determinaron una profunda alteración del comportamiento demográfico en los países implicados que, en poco tiempo, registraron un descenso acusado de la mortalidad, especialmente de la infantil, y un retroceso, igualmente significativo, de la fecundidad, iniciando con ello su proceso de envejecimiento demográfico (Arango, 1987).

En idéntico sentido, el atraso económico de España respecto de los países industrializados, supuso un marcado retraso en la aparición de las alteraciones demográficas que, al fin y a la postre, conducen al envejecimiento demográfico (Nadal, 1975). La batalla definitiva contra la mortalidad catastrófica, que ya se había librado en Europa a lo largo del siglo XVIII, se demoró en nuestro país hasta finales del siglo XIX y comienzos del XX, el retroceso significativo de la mortalidad ordinaria no se produjo hasta el primer tercio de la pasada centuria y la fecundidad no se incorporó a una tendencia descendente hasta comienzos del siglo XX y, aunque tal descenso se acentuó como consecuencia de la Guerra Civil, lo cierto es que después se estancó 
en valores elevados dando lugar al "baby boom" que se registró aproximadamente entre los años 1957 y 1977 (Gil y Cabré, 1997).

Por tales motivos, ese envejecimiento demográfico que ya afectaba y preocupaba a los países más desarrollados iba a tardar aún algún tiempo en hacer acto de presencia en nuestro país. Con una proporción de 36,9 mayores de 65 años por cada 100 jóvenes España era, en 1975, uno de los países menos envejecidos de Europa.

No obstante, en estas fechas el índice de envejecimiento nacional enmascaraba la existencia de acusados contrastes demográficos en el interior del país. Desde la década de los sesenta, las tres cuartas partes del territorio nacional habían registrado saldos migratorios negativos en tanto que sólo un $10 \%$ había logrado arrojar un balance positivo (Puyol, 1988). Obviamente, tanto el impacto inmediato de la emigración como sus posteriores y prolongados efectos, determinaron la existencia de dos realidades demográficas distintas, con diferencias crecientes que sólo el paso del tiempo y la implementación de políticas de cohesión interterritorial han logrado maquillar. Es intención del presente artículo, que Extremadura sirva de ejemplo para ilustrar el surgimiento temprano y traumático proceso de envejecimiento demográfico en las regiones ruralizadas de España, y su progresivo agravamiento hasta niveles que podrían poner en riesgo su futuro socio-económico.

\section{Un proceso de envejecimiento temprano y traumático}

A mediados de los años setenta, el panorama demográfico de Extremadura era ya bien distinto al nacional, de modo que manifestaba claros síntomas de envejecimiento. El índice correspondiente se situaba en un 46,4 por 100 y reflejaba un incremento de más del 80 por 100 en relación con los valores de 1960 (25,7 por 100). Obviamente, el desencadenante del proceso no fue otro que el éxodo que padeció la región entre el inicio de los años sesenta y mediados de los setenta. La pérdida de población adulta y joven propició un incremento proporcional de población vieja que inmediatamente habría de desembocar en una caída vertiginosa de la natalidad y una interrupción de la tendencia descendente que venía mostrando la mortalidad desde los años cuarenta.

Los datos justifican con inusual verosimilitud tales alteraciones. Hasta finales de los años cincuenta, no llegaban a seis mil los extremeños abandonaban anualmente la región. Poco después, la emigración escaló hasta cotas que habrían de empequeñecer sobremanera tales guarismos: entre 1963 y 1965 se registró una salida media anual próxima a las cuarenta mil personas. La menudencia emigratoria de antaño, con 
hombres jóvenes y solteros como principales protagonistas, se trocó en la partida masiva de hombres y mujeres, de niños, jóvenes y adultos (Pérez y Barrientos, 2005).

Como no podía ser de otro modo, tan intensa sangría emigratoria no sólo diezmó la población extremeña, sino que además provocó alteraciones significativas en su estructura por edad y, en relación con ello, un acusado descenso de la natalidad, una tendencia alcista de la mortalidad y, consiguientemente, un debilitamiento severo de los saldos vegetativos. No en vano, el 53,1 por 100 de la población emigrante tenía menos de 25 años, alcanzando una representatividad del 43,5 por 100 los de edades comprendidas entre los 25 y 64 años.

La emigración de las generaciones más jóvenes y fecundas provocó un retroceso de la natalidad que, por primera vez en la historia, alcanzó tasas inferiores a las nacionales en el quinquenio 1966-70. El progresivo envejecimiento demográfico que desde entonces viene padeciendo la región, es una de las causas explicativas de la caída sostenida de su natalidad. Pero también se ha producido una disminución sensible de la fecundidad. En los años cincuenta el número medio de hijos por mujer estaba muy próximo a cuatro, en 1975 se había reducido a 2,65 y en 1998 se situó en 1,25. Los últimos años han registrado una recuperación hasta situarse en 2008 en 1,37 hijos por mujer, inferior pese a todo a los 1,46 de la media nacional y muy por debajo del índice de reemplazamiento generacional. Aunque con un sensible retraso, las ideas, las formas y los comportamientos urbanos van encontrando acomodo en la región y se desdibujan los trazos de ruralidad que tradicionalmente definieron su comportamiento demográfico (Pérez, 2006).

También la mortalidad se hace eco de ese comportamiento asimétrico que muestran otras variables poblacionales a lo largo de la pasada centuria. Una vez más, la década de los sesenta marca una frontera cronológica en el signo evolutivo de sus tasas y resalta la relevancia de la emigración en la demografía extremeña (Barrientos, 1990). Las tasas regionales se situaron en cotas superiores a las medias nacionales y su tendencia descendente se quebró para tomar la orientación alcista que aún mantiene en la actualidad. Obviamente, se trata de la consecuencia lógica de un envejecimiento que no sólo se acomoda al modelo migratorio de los años sesenta y setenta, sino que se debe también al espectacular incremento de la esperanza de vida que se ha conseguido desde comienzos del siglo XX y, de manera especial, en los últimos treinta y cinco años .De la misma manera que se ha producido una convergencia en la fecundidad regional y nacional, la esperanza de vida de los extremeños no ha dejado de escalar posiciones en los últimos tiempos hasta situarse en cotas similares a las del conjunto del país. En 1975 la esperanza de vida de los extremeños apenas superaba mínimamente los 73 años, mientras que en el 2008 dicha edad media se situaba en 80,94 años. En 33 años, la población extremeña ha conseguido una ganancia de vida de 7,9 años y, como el resto del país, se ha situado entre los grupos humanos más longevos del mundo. 


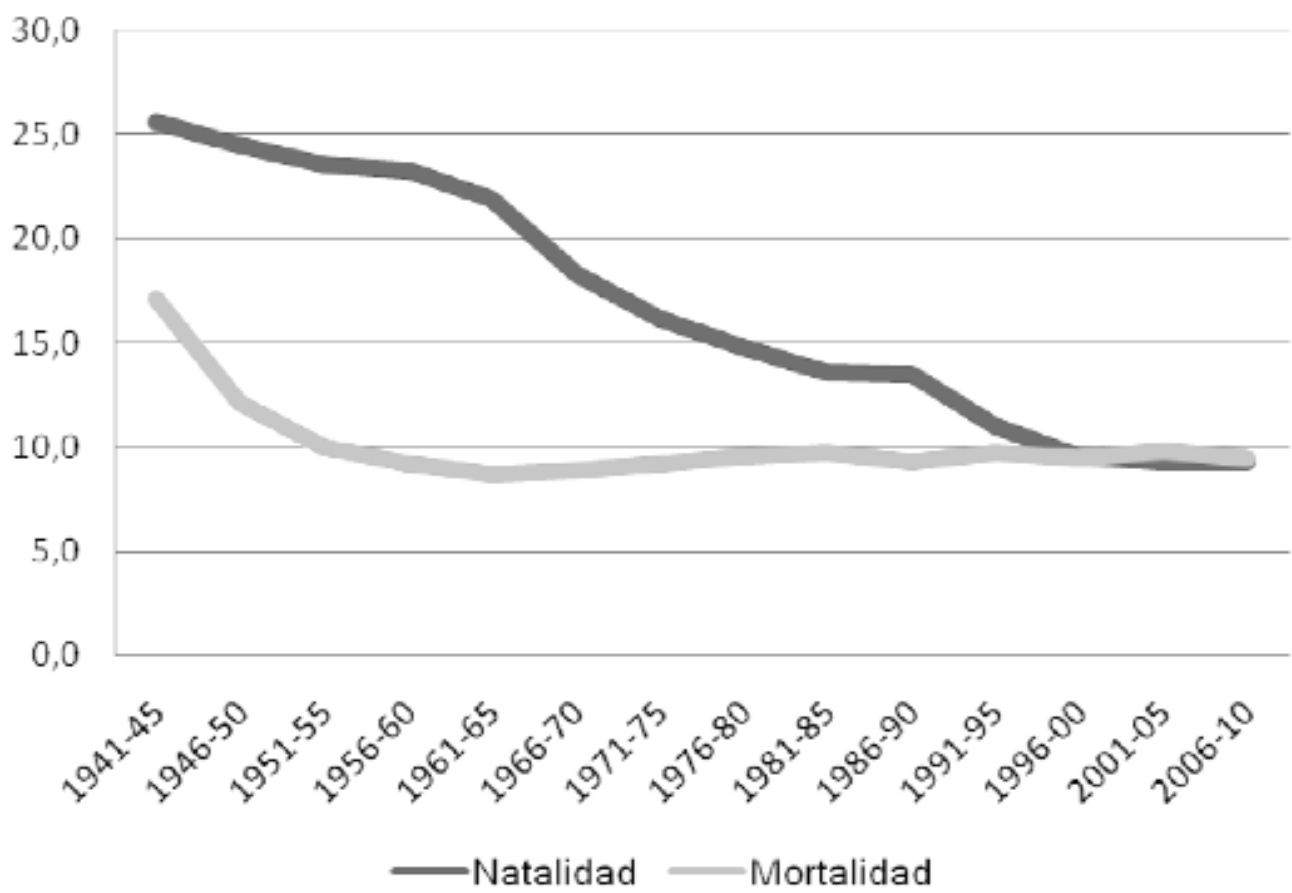

Gráfico 1. Evolución de las variables vegetativas en Extremadura.

\section{Estancamiento demográfico actual}

Según la revisión del Padrón Municipal de Habitantes a 1 de enero de 2010, Extremadura cuenta con un volumen de 1.105.481 habitantes. De ellos, 211.985 superan la edad de 65 años, cifra ésta que representa una participación del 19,2 por 100 sobre el total y que supera en 2,3 puntos porcentuales la media nacional (16,9 por 100). Por contra, el colectivo de personas con menos de 15 años únicamente aglutina a 159.395 jóvenes (15,6 por 100), hecho éste que indica que en la región existe una relación de 133 mayores de 65 años por cada 100 jóvenes con menos de 15 (frente a una media nacional de 114,8).

Para dar idea de la velocidad a la que viene afianzándose este proceso, conviene destacar que en 1991 el volumen de mayores de 65 años era de 162.548 personas, lo que arrojaba una proporción de mayores del 15,3 por 100 frente a una media nacional del 13,8 por 100. En menos de dos décadas, el colectivo de mayores de 65 años 
se ha incrementado en un 30,4 por 100 y, dentro del conjunto, la población octogenaria lo ha hecho en un 73,1 por 100. Obviamente, estos guarismos no hacen más que corroborar la existencia de un proceso de envejecimiento demográfico regional de dimensiones sensiblemente superiores al nacional y que inscribe de lleno a la región en un contexto demográfico moderno al que corresponde una evolución poblacional caracterizada por la atonía, el estancamiento y, en fases avanzadas, la recesión. No en vano, según estimaciones del Instituto Nacional de Estadística (INE, 2010), la población extremeña se situará en 2020 en 1.086.024 habitantes lo que, de producirse, representaría una pérdida del 1,8 por 100 respecto de la población registrada en 2010.

En lo que concierne a algunas de sus características estructurales, la fisonomía de la pirámide del año 2010, destaca por la existencia de una base muy estrecha y simétrica que refleja la consolidación de un proceso de desfecundidad de dimensiones similares a las del conjunto nacional, al tiempo que evidencia la escasa incidencia que ha tenido la inmigración extranjera sobre dicha variable, lo que constituye un factor diferenciador con respecto a la evolución reciente de la natalidad tanto en el conjunto del país como en algunas regiones de características socioeconómicas comparables a Extremadura. (Martínez, Gil y Gómez, 2008). Similar consideración cabe realizar sobre la población con edades comprendidas entre los 15 y 30 años, aunque en este caso la incipiente caída de la fecundidad se solapaba con la desnatalidad que había propiciado el éxodo rural de los años sesenta y setenta.

Entre los 30 y 50 años, y tanto en el lado de los hombres como en el de las mujeres, la pirámide muestra un destacado ensanchamiento que representa al grueso del "baby boom" español, unas generaciones que aglutinan a 337.281 personas y representan el 30,5 por 100 de la población extremeña (Castro, 2000). Se trata, en gran medida, de los extremeños que consiguieron esquivar los embates de la emigración, que constituyen el grueso de la fuerza laboral de la región, que conviven con unos hijos que abandonan el hogar cada vez más tarde y con unos padres más longevos, y que asisten también, con ostensible expectación, a un bombardeo informativo que siembra de inquietud y de zozobra sus proyectos de futuro justo en el momento en que se aproximan a la edad de jubilación. Ello se producirá a partir, aproximadamente, del año 2020 y se prevé que esta circunstancia habrá de resultar tan comprometida como lo fue su paso por el sistema educativo o su incorporación al mundo laboral, que disparó las tasas de desempleo hacia cotas hasta entonces inéditas: en otras palabras, la jubilación de las generaciones del baby boom va a ejercer una presión importante sobre los sistemas de protección social, sobre los servicios de atención sociosanitaria y, cómo no, también sobre las pensiones (Abellán y Puga, 2005).

A partir de los cincuenta años se hace patente el vaciamiento provocado por la intensa emigración padecida en Extremadura entre el inicio de los años sesenta y mediados de la década de los setenta, que ha sangrado las cohortes correspondien- 


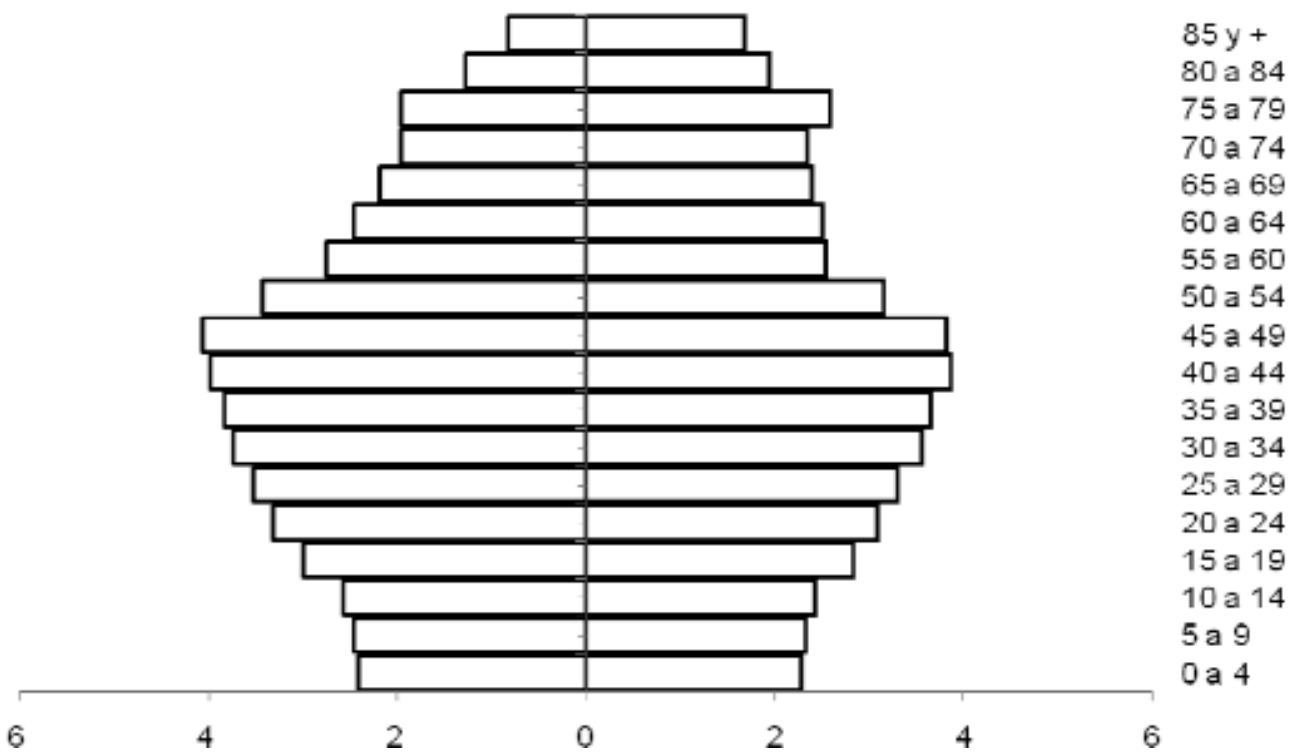

Gráfico 2. Pirámide de Edades. Extremadura, 2010.

tes a los 50-70 años provocando unas entalladuras prácticamente simétricas y reduciendo la participación de este colectivo al 21,4 por 100 del total.

Por último, coronando la pirámide, se sitúa ese nutrido grupo de mayores de 65 años que, como ya se ha dicho, alcanza una proporción del 19,2 por 100, y que refleja convenientemente ese incremento de la esperanza de vida que, junto con el retroceso de la fecundidad, explica el proceso de envejecimiento demográfico referido.

Estas peculiaridades estructurales, la convergencia de las variables vegetativas y la consiguiente atonía que manifiesta actualmente el crecimiento natural, parecen sugerir que serán las migraciones las que, manteniendo el protagonismo de otros tiempos, vayan a condicionar en mayor medida el futuro demográfico extremeño. Conviene destacar, a este respecto, dos circunstancias que definen la dinámica espacial de los últimos años:

En primer lugar, debe significarse que el comportamiento migratorio de los extremeños aparece sumido en un ambiente de indecisión que cristaliza en la alternancia de períodos con balances migratorios de signo cambiante. En épocas de bonanza económica el saldo migratorio muestra un signo negativo, en tanto que en momentos de crisis o de recesión, el balance es positivo para Extremadura. Podría aseverarse, 
en tal sentido, que en el medio plazo se produce una neutralización de los flujos de entrada y salida, lo que limita el alcance de estos movimientos sobre la demografía regional.

En segundo lugar, y en estrecha relación con lo anterior, existe una similitud estructural entre la corriente emigratoria y la de inmigración y retorno, con lo que los desajustes estructurales que provoca la primera se ven parcial o totalmente corregidos por los efectos positivos que genera la llegada de antiguos emigrantes y de población foránea. Las migraciones registradas entre 2002 y 2008 constituyen un ejemplo inigualable para valorar estas circunstancias.

Tabla 1. Migraciones recientes en Extremadura.

\begin{tabular}{|c|c|c|c|}
\hline Años & Bajas & Altas & Saldo \\
\hline 2002 & 10.568 & 7.734 & -2.834 \\
2003 & 10.466 & 9.020 & -1.446 \\
2004 & 10.131 & 8.951 & -1.180 \\
2005 & 10.008 & 8.745 & -1.263 \\
2006 & 11.179 & 10.740 & -439 \\
2007 & 12.083 & 12.350 & 267 \\
2008 & 13.298 & 18.022 & 4.724 \\
\cline { 2 - 4 } Total & 77.733 & 75.562 & -2.171 \\
\hline
\end{tabular}

En el período de referencia Extremadura ha contabilizado la salida, con destino a otras comunidades autónomas y al extranjero, de 77.733 personas. En el mismo tiempo se registraron en la región un total de 75.562 altas correspondientes a inmigrantes, nacionales y extranjeros, y a retornados. El balance migratorio, por tanto, arroja un débil saldo negativo de 2.171 individuos en siete años. Ambos flujos, pues, mantienen un comportamiento especialmente dependiente de la coyuntura económica nacional, como lo demuestra el saldo migratorio de los años 2007 y 2008, y por tanto unas alternancias que provocan que, en el medio plazo al menos, el volumen de entradas y salidas se sitúe en cotas próximas. En cualquier caso, debe convenirse que las migraciones regionales no constituyen fenómenos exangües, sino que prevalecen como fenómenos activos cuyas repercusiones no solamente se manifiestan en la evolución de la población absoluta, sino también en sus características estructurales y, lo que es aún más importante, en su distribución espacial.

En lo que concierne a los rasgos estructurales de estos flujos migratorios, la corriente emigratoria aparece caracterizada por un marcado equilibrio en el reparto por sexos y por una distribución por edades que permite comprobar que el 59,8 por 
100 del colectivo se encuadra entre los 20 y 44 años de edad, seguido en importancia por los menores de 20 años cuya participación se sitúa en un 16,3 por 100. Por su parte, el grupo de población adulta-vieja sólo logra una representatividad del 12,9 por 100, correspondiendo la menor proporción, como era de esperar, a los mayores de 65 años que, a pesar de todo, representan el 11 por 100 de la población emigrante.

Lejos de la idea que habitualmente se tiene de la inmigración y el retorno, tanto la distribución por sexos como por edades son muy similares a la del flujo negativo. En el primer caso, se registra una mayor presencia masculina $(54,1$ por 100$)$ que debe vincularse sobre todo a la mayor participación de los varones en la inmigración y el retorno de extremeños procedentes del extranjero. En lo relacionado con el reparto por edades, un 20,7 por 100 de las altas se han correspondido con menores de 20 años y un 56,7 por 100 han sido cursadas por personas con edades entre 20 y 44 años. La tercera posición la ocupa el grupo de población adulta-vieja, con un 15,8 por $100 \mathrm{y}$, a bastante distancia, se sitúa el colectivo de mayores con una participación del 6,8 por 100 sobre el total de altas contabilizadas en la región (Pérez y Barrientos, 2005).

En función de esta similitud cuantitativa y estructural de los flujos migratorios, no cabe duda de que la afluencia de inmigrantes y retornados está representando una bocanada de aire fresco que contribuye a oxigenar el tejido demográfico extremeño. No en vano, el aporte de población joven y adulta supera con creces al de población vieja, desterrando con ello la frecuente apreciación de que Extremadura, como otros espacios de la España interior, sólo adquiere una cierta relevancia como destino de retorno de sus antiguos emigrantes jubilados.

\section{Perspectivas de futuro}

La convergencia de las variables vegetativas y la semejanza que gobierna la dinámica espacial de los extremeños desde la primera mitad de los años ochenta, permiten comprender la situación de estancamiento que viene padeciendo la población regional en las tres últimas décadas y que, con toda probabilidad, seguirá sufriendo a medio y largo plazo. Es cierto que, como ha ocurrido a escala nacional, estos condicionamientos podrían verse alterados por la afluencia de inmigrantes y sus consiguientes efectos sobre la fecundidad. Ni la situación del mercado laboral ni la actual coyuntura económica hacen verosímil, empero, que Extremadura pueda atraer y fijar un volumen de población extranjera muy superior a los 38.747 contabilizados en 2010. Y, aunque así fuera, su distribución espacial estaría muy localizada y sus efectos se reducirían a unos pocos enclaves que son, precisamente, los que menos problemas demográficos acusan. 
Las proyecciones del Instituto Nacional de Estadística no hacen sino confirmar esta hipótesis (INE, 2010). Partiendo de la Estimación de Población Actual a 1 de enero y de hipótesis de evolución de cada uno de los componentes demográficos básicos, la proyección obtiene para 2020 una población de 1.086.024 habitantes, cifra ésta que apenas representa una evolución positiva del 0,3 por 100 en relación con la población estimada en 2010.

Tabla 2. Proyección de la población extremeña.

\begin{tabular}{|c|c|c|}
\hline Años & Población total & $2010=100$ \\
\hline 2010 & 1.082 .796 & 100,0 \\
2011 & 1.083 .709 & 100,1 \\
2012 & 1.084 .335 & 100,1 \\
2013 & 1.084 .885 & 100,2 \\
2014 & 1.085 .345 & 100,2 \\
2015 & 1.085 .709 & 100,3 \\
2016 & 1.085 .967 & 100,3 \\
2017 & 1.086 .120 & 100,3 \\
2018 & 1.086 .173 & 100,3 \\
2019 & 1.086 .137 & 100,3 \\
2020 & 1.086 .024 & 100,3 \\
\hline
\end{tabular}

Tras dicho estancamiento poblacional subyacería un retroceso del 17 por 100 en el número de nacimientos y un aumento del 0,1 por 100 en el volumen de defunciones, lo que representaría una caída de la natalidad a valores del 8 por 1.000 en 2019 y un estancamiento de la mortalidad en cotas del 10 por 1.000 , con el subsiguiente balance negativo para un Crecimiento Natural que se situaría en un -2,1 por 1.000 .

Tabla 3. Proyección de las variables vegetativas.

\begin{tabular}{|c|c|c|c|}
\hline Años & Tasa Nat. & Tasa Mort. & Crec. Nat. \\
\hline 2010 & 9,6 & 10,0 & $-0,4$ \\
2011 & 9,3 & 10,0 & $-0,7$ \\
2012 & 9,2 & 10,0 & $-0,8$ \\
2013 & 9,0 & 10,0 & $-1,0$ \\
2014 & 8,9 & 10,0 & $-1,2$ \\
2015 & 8,7 & 10,1 & $-1,4$ \\
2016 & 8,5 & 10,1 & $-1,5$ \\
2017 & 8,3 & 10,1 & $-1,7$ \\
2018 & 8,1 & 10,1 & $-1,7$ \\
2019 & 8,0 & 10,0 & $-2,1$ \\
\hline
\end{tabular}


En el ámbito migratorio, se prevé la existencia de saldos positivos con tendencia alcista, de modo que se pasaría de un diferencial positivo de 1.300 altas en 2010, a un balance positivo de 2.137 migrantes en 2.019. En cualquier caso, ambos flujos migratorios continuarían situándose en cotas muy similares, especialmente en lo concerniente a los movimientos con origen y destino nacional, y sólo se registrarían diferencias moderadas entre el volumen de altas y bajas cursados desde o hacia el extranjero, respectivamente.

Tabla 4. Proyección de las migraciones extremeñas.

\begin{tabular}{|c|c|c|c|c|}
\hline 2010 & $\begin{array}{c}\text { Inmigración } \\
\text { Nacional }\end{array}$ & $\begin{array}{c}\text { Inmigración } \\
\text { Extranjera }\end{array}$ & $\begin{array}{c}\text { Emigración } \\
\text { Nacional }\end{array}$ & $\begin{array}{c}\text { Emigración } \\
\text { Extranjera }\end{array}$ \\
\hline 2010 & 16.825 & 3.748 & 16.719 & 2.554 \\
2011 & 16.794 & 3.748 & 16.622 & 2.541 \\
2012 & 16.755 & 3.748 & 16.514 & 2.526 \\
2013 & 16.711 & 3.748 & 16.399 & 2.508 \\
2014 & 16.663 & 3.748 & 16.279 & 2.489 \\
2015 & 16.610 & 3.748 & 16.153 & 2.468 \\
2016 & 16.557 & 3.748 & 16.024 & 2.446 \\
2017 & 16.503 & 3.748 & 15.894 & 2.423 \\
2018 & 16.452 & 3.748 & 15.764 & 2.400 \\
2019 & 16.402 & 3.748 & 15.636 & 2.377 \\
\hline
\end{tabular}

En líneas generales, por tanto, en el año 2020 Extremadura continuaría caracterizándose por un estancamiento poblacional que se sustentaría sobre las bases de un progresivo debilitamiento de la natalidad y una estabilización de la mortalidad que desembocaría en un saldo natural negativo de 2.251 personas, déficit éste que se vería parcialmente compensado por la existencia de un balance migratorio positivo de 2.137 personas.

Evidentemente, este comportamiento de las variables demográficas propicia unas alteraciones estructurales que se manifiestan de forma nítida en la distribución por edades. Como es previsible en función de la evolución demográfica extremeña de los últimos treinta años, la población regional camina inexorablemente hacia una intensificación del proceso de envejecimiento demográfico. Esta situación, que viene propiciada por el retroceso paulatino de la natalidad y el aumento de la esperanza de vida, se concretará en una reducción del contingente poblacional con edades inferiores a 45 años, un aumento significativo de la población adulta-vieja y un incremento no menos sensible de los mayores de 65 años.

Debe significarse que, dentro del colectivo de mayores, la población octogenaria crecerá en un 15,3 por 100 , llegando a 71.271 la cifra de habitantes que superarán 
Tabla 5. Estructura por edad de la población extremeña.

\begin{tabular}{|c|c|c|c|}
\hline Edad & 2010 & 2020 & $2020 / 2010(\%)$ \\
\hline$>15$ & 157.622 & 155.399 & $-1,4$ \\
$15-44$ & 457.677 & 396.270 & $-13,4$ \\
$45-64$ & 263.794 & 318.646 & 20,8 \\
$>65$ & 203.707 & 215.703 & 5,9 \\
$>80$ & 61.827 & 71.271 & 15,3 \\
I. Envejecimiento & 129,2 & 138.8 & 7,4 \\
\hline
\end{tabular}

esta edad. Es innegable, en consecuencia, que los retos que plantea el envejecimiento demográfico regional van a acrecentarse como consecuencia de las mayores atenciones que requiere este colectivo de octogenarios, y los mayores costes que implicará la superior demanda de servicios sanitarios y sociales. A ello se añade, como anteriormente se indicó, que a partir de 2020 llegarán a la edad de jubilación las nutridas generaciones del baby-boom español. Como ya comienza a ocurrir en no pocos hogares extremeños, en un futuro próximo muchas parejas maduras tendrán a su cargo menos hijos que padres, circunstancia ésta de la que se derivará una serie de repercusiones familiares, afectivas y socioeconómicas de innegable trascendencia. Compartimos plenamente la opinión de quienes consideran que el crecimiento de los octogenarios debe valorarse positivamente por cuanto representa un triunfo demográfico, social y científico en la lucha contra la muerte (Pérez, 2005). Sin embargo, por optimista que sea la perspectiva, no debemos infravalorar la incidencia que tal circunstancia tendrá en la demanda de servicios de atención sociosanitaria, en el consumo de medicamentos, en la adecuación de las viviendas, en la adaptación de los transportes o en el requerimiento de profesionales especializados en el cuidado de los muy mayores.

\section{Bibliografía}

Abellán García, A.-Puga González, Maㅗ. D. (2005). Una España que envejece. Papeles de Economía Española, 104, 57-75.

Arango, J. (1987). La modernización demográfica de la sociedad española. En Nadal, J. et al. La economía española en el siglo XX. Una perspectiva histórica, Barcelona, Ariel, pp. 201-236.
Barrientos Alfageme, G. (1990). Geografía de Extremadura. Badajoz, Universitas Editorial

Castro, T. (2000). Un caso especial: la generación del baby-boom. En Castiello Ma ${ }^{2}$ T. (coord.). Las personas mayores en España. Madrid, Imserso, 101-108.

Gil Alonso, F.-Cabré, A. (1997). El crecimiento natural de la población española y sus 
determinantes. En Puyol, R. (edt.) Dinámica de la población en España. Madrid, Síntesis, pp. 47-144.

Macarrón Larrumbe, A. (2010). El suicidio demográfico de España. Libertad Digital, 16 de julio.

Martínez, R.-Gil, E.-Gómez, J. M. (2008). La inmigración en el poblamiento de la región de Murcia. Universidad de Murcia.

Nadal, J. (1975). El fracaso de la revolución industrial en España. Barcelona, Ariel.

Pérez Díaz, A.-Barrientos Alfageme, G. (2005). Emigrantes, inmigrantes y retornados. Extremadura ante el siglo XXI. Badajoz,
Servicio de Publicaciones de la Excma. Diputación Provincial.

Pérez Díaz, A. (2006). Los pequeños municipios ante los retos del desarrollo. Norba. Revista de Geografia, XI, 183-197.

Pérez Díaz, A. (2008). La inmigración de retorno a Extremadura. Revista de Estudios Extremeños, 1331-1364.

Pérez Díaz, J. (2005). Consecuencias sociales del envejecimiento demográfico, Papeles de Economía Española, 104, 210-226.

Puyol, R. (1988). La población española. Madrid, Síntesis. 